\title{
¿Jaque virtual a la relación forma-contenido en la enseñanza?
}

\section{Virtual check to the form-content relationship in teaching?}

\section{Celia Salit}

https://orcid.org/0000-0002-7816-4836

celiasalit2112@gmail.com

Facultad de Artes y de FFYH. UNC. I Argentina

\section{Jennifer Cargnelutti}

https://orcid.org/0000-0002-4629-3983

jennifercargnelutti@gmail.com

Facultad de Artes y de Ciencias Económicas.

UNC. Argentina

\author{
Marina Yazyi \\ https://orcid.org/0000-0002-6890-5768 \\ marina.yazyi@gmail.com \\ Facultad de Artes y de FFYH. UNC. I Argentina
}

\author{
Ana Belén Caminos \\ https://orcid.org/0000-0002-3604-7922 \\ anabelencaminos@gmail.com \\ Facultad de Artes y de FFYH. UNC. Argentina
}

\section{RESUMEN}

Frente a las particulares circunstancias que vivimos producto del aislamiento social preventivo y obligatorio, un conjunto de interrogantes se nos plantean como formadoras de docentes, integrantes del equipo de la cátedra de Didáctica General de la Facultad de Artes de la UNC, ¿cómo se piensa la mediación y la intervención en las aulas virtuales? ¿Cómo se acompaña a les estudiantes en el cursado con esta modalidad? ¿Cómo construir propuestas de enseñanza virtuales que articulen forma y contenido en la enseñanza? ¿Qué otros desafíos didácticos plantea la enseñanza en la virtualidad? Ante estos interrogantes, procuramos acercar en esta presentación una breve fundamentación teórica acerca de la relación forma-contenido, sentidos y modos de concreción que se le imprime en las clases presenciales, una referencia a los desafíos que se nos plantean en el marco de la actual situación, en vínculo con algunas de las opciones asumidas en la intención de sostener la articulación forma-contenido en la virtualidad, en torno a: el tiempo/los tiempos y el espacio; la escritura, el acompañamiento/seguimiento de les estudiantes.

\section{ABSTRACT}

Faced with the particular circumstances we are experiencing as a result of preventive and compulsory social isolation, a series of questions are posed to us as teacher educators, members of the team of the Chair of General Didactics of the UNC Faculty of Arts, how do you think the mediation and intervention in virtual classrooms? How are students accompanied in the course with this modality? How to build virtual teaching proposals that articulate form and content in teaching? What other didactic challenges does teaching in virtuality pose? Faced with these questions, we try to bring together in this presentation a brief theoretical foundation about the relationship between form-content, meanings and modes of concretion that are imprinted in the face-to-face classes, a reference to the challenges that we face in the framework of the current situation, in connection with some of the options assumed in the intention of sustaining the form-content articulation in virtuality, around: time / times and space; writing, accompaniment / monitoring of students.

PALABRAS CLAVE enseñanza, virtualidad, forma, contenido

\section{KEY WORDS}

teaching, virtuality, form, content 


\title{
1.PRESENTACIÓN
}

\author{
Las posibilidades de internet y \\ de la informatización tecnológica \\ de la enseñanza cultivan la ilusión \\ de la exclusión del cuerpo erótico y de la \\ transferencia de la relación de la enseñanza. \\ Es lo que Ricardo Massa define como \\ "falacia de la tecnología didáctica". \\ Recalcati, M. 2016
}

Como formadoras de docentes, más aún como equipo de una cátedra de Didáctica General, la enseñanza se configura en núcleo, cuestión central que atraviesa nuestras prácticas. Prácticas que sostenemos en la convicción de que se "enseña a enseñar enseñando".

En consonancia con ello, nos proponemos atender en los encuentros con les estudiantes a la relación entre las dos dimensiones clave de la enseñanza: la forma y el contenido. Relación que opera como eje teórico metodológico de la propuesta de la cátedra y que procuramos poner en acto en cada una de las clases. En efecto, construir para cada núcleo conceptual propuestas que atiendan a la articulación forma-contenido es y ha sido siempre el principal desafío para nuestras prácticas.

El presente, atravesado por la expansión de la pandemia provocada por el Covid-19, nos enfrenta a condiciones de vida y de trabajo inusuales, regidas por el aislamiento obligatorio que -entre otras medidas- impuso la suspensión de clases presenciales. Esta situación nos compele a reemplazar la interacción cara a cara con el otro/los otros, por una comunicación mediada por la tecnología digital.

Esta posibilidad nos es dada puesto que la Facultad de Artes de la Universidad Nacional de Córdoba -institución de pertenencia- cuenta con una plataforma Moodle donde se radican las aulas virtuales de los diferentes espacios curriculares de las carreras que ofrece. A su vez, la facultad cuenta con un Área Tecnología Educativa, cuya labor de acompañamiento a las propuestas presenciales desde espacios virtuales se viene impulsando en cumplimiento tanto de las políticas universitarias como las de la propia unidad académica. Hoy las aulas virtuales han tomado protagonismo central dado que es el único espacio para garantizar el cursado de les estudiantes.

Si bien contar con el espacio virtual de trabajo nos permite la continuidad pedagógica en el marco de la pandemia, el nuevo escenario nos interpela particularmente, ¿cómo construir propuestas de enseñanza virtuales que atiendan a esta relación? ¿Cuáles son las formas? ¿Cómo se piensa la mediación y la intervención en las aulas virtuales? 
¿Cómo se acompaña a les estudiantes en el cursado con esta modalidad? ¿Qué otros desafíos didácticos plantea la enseñanza en la virtualidad? ¿Qué modos de resolución venimos construyendo?

¿Cómo se piensa la mediación y la intervención en las aulas virtuales? ¿Cómo se acompaña a les estudiantes en el cursado con esta modalidad? ¿Qué otros desafíos didácticos plantea la enseñanza en la virtualidad? ¿Qué modos de resolución venimos construyendo?

Ante los interrogantes, procuramos acercar en esta presentación, una breve fundamentación teórica acerca de la relación forma-contenido, sentidos y modos de concreción que se le imprime en las clases presenciales, una referencia a los desafíos que se nos plantea el marco de la actual situación, en vínculo con algunas de las opciones asumidas en la intención de sostener la articulación forma-contenido en la virtualidad, en torno a: el tiempo/los tiempos; el espacio; la escritura, el acompañamiento/seguimiento de les estudiantes, componentes operativos de toda construcción metodológica.

\section{A. ALGUNAS PRECISIONES CONCEPTUALES ACERCA DE LA RELACIÓN FORMA-CONTENIDO EN LA ENSEÑANZA}

Enseñar siempre es un desafío y conlleva exponer y exponerse. Básicamente consiste en disponer las mejores condiciones para incluir al otro en eso que llamamos "cultura". Pero cuando se trata de enseñar acerca de la enseñanza a otros que enseñarán, la tarea asume unas características específicas que ponen a prueba los propios modos de enseñar de quienes nos dedicamos a la formación docente. Ello dado que, se teoriza acerca de la enseñanza mientras se enseña.

En otros términos, sostenemos que se enseña -y se aprende- desde lo que Edwards caracteriza como la lógica del contenido y la lógica de la forma. Con la primera refiere a aquello que (...) involucra los presupuestos epistemológicos desde los cuales los conocimientos han sido formalizados. La segunda remite a la dinámica en la cual se concretiza la presentación-apropiación del conocimiento, a los sentidos que se objetivan en los modos en que maestros y alumnos se vinculan entre sí e incluye tanto el discurso explícito como lo implícito en los sujetos. (Edelstein, Salit, Domjan, Gabbarini, 2014, p. 4)

En consonancia con Edwards, sostenemos como principio estructurante de nuestras prácticas que: "El contenido no es independiente de la forma en la cual es presentado. La forma tiene significados que se agregan al "contenido" transmitido produciéndose una síntesis, un nuevo contenido". (1997, p. 47) 
En consonancia con Edwards, sostenemos como principio estructurante de nuestras prácticas que: "El contenido no es independiente de la forma en la cual es presentado. La forma tiene significados que se agregan al "contenido" transmitido produciéndose una síntesis, un nuevo contenido". (1997, p. 47)

En clave con esta postulación, consideramos en otro texto de nuestra autoría que en algunos tramos o en relación con algunas temáticas de la formación docente, se plantea una particular situación en la cual parecieran borrarse las brechas entre conocimiento disciplinar y conocimiento pedagógico.

Ahora bien, en la línea de aportar ciertas precisiones al campo de la Didáctica General, le asignamos al concepto forma un alcance que excede al planteo de Verónica Edwards. Desde nuestra visión, este no se reduce a la "presentación del contenido" sino que implica un conjunto de maneras de hacer y de decir al enseñar mientras se enseña, no siempre conscientemente anticipadas, diseñadas; no siempre conscientemente puestas en juego durante la acción en el aula.

En la misma línea, en otro trabajo, afirmamos que:

\begin{abstract}
(...) lo que contiene la forma es más que ideas, sentimientos y percepciones; en la forma está también el contenido, el modo mismo de concebirlo, de darle sentido, de expresarlo, de hacerlo visible. La forma es una manera de hacer las cosas, de ver y decir; de hacer ver y hacer decir no sólo da cauce sino que es materialización, expresión de lo sensible y lo decible. (Salit, 2011)
\end{abstract}

En síntesis, no es posible abstraer el contenido de la forma, son dos dimensiones a entramar necesariamente en la enseñanza. Entramado a atender especialmente si se trata de guardar coherencia entre aquello que uno dice acerca de la enseñanza y lo que se enseña sobre ella desde los modos concretos de intervenir. Ello requiere ejercer una fuerte vigilancia si se quiere evitar el riesgo de que se produzca un quiebre, una contradicción.

Sin duda, la idea de que "la forma es contenido y que el contenido se transforma en la forma" es y ha sido una expresión potente que marca nuestras maneras de pensar la enseñanza y, en consecuencia, de construir nuestras propuestas de formación, tradicionalmente, de carácter presencial, en el ámbito del aula universitaria.

Para mostrar el modo en que ponemos "en práctica" en la presencialidad este posicionamiento teórico/metodológico, describimos a continuación una clase que consideramos central en el desarrollo de la materia y que colaborará luego en la comprensión de los sentidos que intentamos desplegar en la virtualidad. 


\section{B. CLASE PRESENCIAL "FORO: EL ARTE Y SU ENSEÑANZA"}

Hace tiempo venimos implementando presencialmente en nuestras prácticas de enseñanza una clase que consideramos paradigmática. En ella se tematiza la relación forma-contenido en la enseñanza, y se incorporan dos ideas fuerza que forman parte de los contenidos de la propuesta y que operan a modo de "contenido excusa": una relativa a los aportes de Jerome Bruner para la comprensión del aprendizaje y otra en vínculo con la especificidad de los trayectos formativos de nuestros estudiantes, futuros docentes en Artes Visuales, Educación Musical y Teatro.

\section{Hace tiempo venimos implementando presencialmente en nuestras prácticas de enseñanza una clase que consideramos paradigmática. En ella se tematiza la relación forma-contenido en la enseñanza}

La clase que identificamos como "foro" juega con la idea de "una clase dentro de otra clase", lo que se podría graficar del siguiente modo:

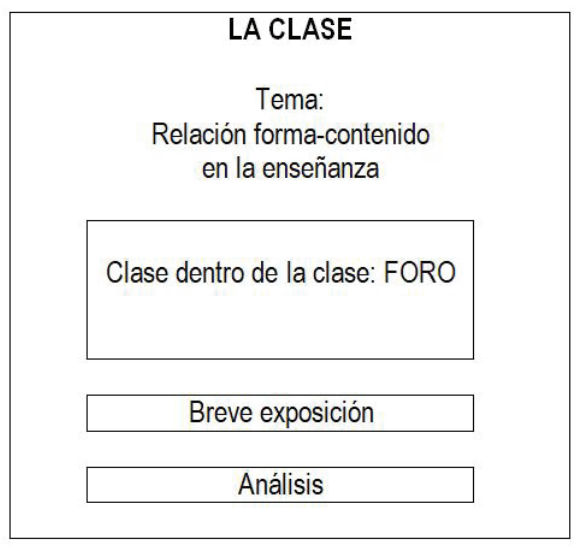

Es importante aclarar que una condición es atender a una secuencia tal que en el encuentro previo al que estamos narrando se desarrollen aportes teóricos del constructivismo, entre ellos los de Bruner, ya que operan a modo de sostén indispensable de la construcción metodológica prevista para esta clase. En el punto de partida, se recuperan dichos aportes desde la lectura de los siguientes párrafos del libro El conocimiento compartido de Edwards y Mercer:

De esta visión de la cultura como foro se desprende que la inducción a la cultura a través de la educación debe también participar del espíritu del foro, de la negociación, de la recreación del significado, si se quiere preparar a los jóvenes para la vida real. (1986, p. 123, 1994, p. 182) 
(...) si se quiere que sea una invitación a la reflexión y a la creación de cultura, el lenguaje de la enseñanza, no puede ser el llamado "lenguaje no contaminado" del hecho y la "objetividad". Debe expresar postura y contrapostura, y, en el proceso, dejar lugar para la reflexión, para la metacognición. Este proceso de objetivar en lenguaje o en imagen lo que se ha pensado y luego darle la espalda y reconsiderarlo es lo que nos permite avanzar. (1986, p. 129, 1994, p. 183)

Una vez compartida las ideas de Bruner se invita al alumnado, en línea con la propuesta del autor, a participar en un "foro" acerca del "Arte y su enseñanza". Se explica luego la dinámica que consiste en "cada grupo recibe una afirmación referida al tema del foro que debe asumir como propia, aún a pesar de que no coincida con ella y elaborar argumentos para defenderla y contraargumentos para confrontar otras posiciones opuestas. Se procede posteriormente a solicitarle a les estudiantes que conformen un número de grupos que debe coincidir con el total de "sentencias" preparadas para la actividad de, aproximadamente, seis integrantes cada uno.

En el armado de las respectivas afirmaciones se tiene en cuenta la existencia de "pares opuestos". De ello resultan las siguientes: -Las artes son un lujo, un saber inútil. Por lo tanto enseñar arte también lo es-; -Las artes son sólo saberes "útiles" para la vida cotidiana de todos los sujetos. Por lo tanto su enseñanza debe dar cuenta de dicha utilidad-; -Las artes tienen únicamente una función instrumental. Por lo tanto su enseñanza no es de carácter formativo-; -Las artes desempeñan un papel importante en el desarrollo de sujetos críticos por lo tanto, en la enseñanza de las mismas, la formación "crítica" es su único objetivo-; -Las artes son fundamentalmente "expresión libre". Por lo tanto, en las clases de arte cada alumno deberá hacer sólo lo que quiere, cuando quiere y como puede-; -Una expresión artística es mera reproducción de otra. Por lo tanto, para la enseñanza de cualquiera de sus disciplinas es necesario hacerlo a partir de la copia de "él" modelo-; -Las artes son disciplinas que deben ocupar un lugar central en el currículo escolar ya que la enseñanza y el aprendizaje de la creatividad y la expresión artística son más importantes que el conocimiento científico-; -Las artes no tienen la misma jerarquía que "las ciencias". Por lo tanto, no deben estar en igualdad de condiciones que el resto de las disciplinas en el currículo escolar. Son sólo materias secundarias-.

Una vez transcurrido el tiempo destinado para ello, se da por iniciado el foro, invitando a participar en él a todos los grupos con la consigna de asumir empáticamente la posición asignada, argumentar y contraargumentar.

Transcurrida esta instancia, finaliza el foro y se propone analizar la clase. Inicialmente se pregunta acerca de cuál es el tema abordado en ella. Algunos estudiantes consideran que son los postulados de Bru- 
ner, otros que es el "Arte y su enseñanza". La profesora devela que es "la relación forma-contenido en la enseñanza" y explica brevemente las ideas de Verónica Edwards que compartimos en el apartado anterior. Reconocimiento que genera una suerte de disrupción prevista en les estudiantes en la idea de desestructurar $y$, a la vez, movilizar un nuevo modo de pensar la enseñanza. Luego, la docente vuelve a proponer que se analice la clase "foro" (clase dentro de la clase) en función de la pregunta acerca de qué modo particular se articulan forma y contenido en ella.

A partir de esta clase, les estudiantes en general, "juegan el juego que se juega" y queda instalada la expectativa, a la vez compromiso para el equipo, para atender a la articulación forma-contenido el resto del cursado y habilitar el espacio para el análisis de cada una de las clases que se suceden.

A partir de esta clase, les estudiantes en general, "juegan el juego que se juega" y queda instalada la expectativa, a la vez compromiso para el equipo, para atender a la articulación forma-contenido el resto del cursado y habilitar el espacio para el análisis de cada una de las clases que se suceden.

Sin embargo, las condiciones de planificación de las clases de Didáctica General, como señalamos, cambiaron abruptamente. Nos encontramos imposibilitados de asistir a clases presenciales. No obstante, se nos informa que es clave en estas circunstancias avanzar en el abordaje de los contenidos disciplinares de cada espacio curricular a los efectos de garantizar, en la medida de lo posible, el cumplimiento del calendario académico previsto. Por ello se hizo necesario disponernos a enseñar en la virtualidad. La pregunta que necesariamente se instala, en este caso, es: ¿Cómo enseñar a enseñar articulando forma y contenido en la virtualidad?

La pregunta que necesariamente se instala, en este caso, es: ¿Cómo enseñar a enseñar articulando forma y contenido en la virtualidad? .

\section{A. PRIMER DESAFÍO.“DAR LA CLASE” EN EL AULA VIRTUAL: LA CONSTRUCCIÓN DE OTROS TIEMPO/S Y ESPACIOS}

Tiempo y espacio dos dimensiones profusamente analizadas desde diversas disciplinas del campo de las Ciencias Sociales y de las Humanidades son, a nuestro entender, también objeto de reflexión de la Didáctica y necesariamente deben incluirse en eso que Litwin (1995) ha dado en Ilamar "la nueva agenda" en tanto se configuran como las dos coordenadas de una clase, por lo cual ya no es posible pensar prácticas de enseñanza en las que no tomen un particular protagonismo. 
Por ello, atender a estas dos categorías forma parte del conjunto de decisiones a asumir en el diseño de una clase.

En congruencia con la preocupación de algunos cientistas sociales, los tiempos de la escolaridad han estado en la mira de quienes se proponen develar las lógicas que subyacen a las dinámicas que la escuela les imprimen. Así, autores como Hargreaves, en Canadá y Terigi, en Argentina, develan que desde la racionalidad tecnocrática se "coloniza" el tiempo con vistas al logro de ciertos fines educativos $y$, se considera, al decir de Poggi (1999), como una variable objetiva, una condición instrumental, que se presenta desde un modelo normalizado, inmutable, administrativamente regulado. Racionalidad que le asigna a los procesos escolares un funcionamiento uniforme $y$, a la vez, fragmentado que Terigi (2008) caracteriza como "cronosistema", es decir, un ordenamiento que establece etapas de la vida para ir a ciertos niveles escolares, ritmos de adquisición de los aprendizajes, duración de las jornadas escolares, duración de la hora, módulo o bloque escolar, etc., desde el presupuesto de "aprendizajes monocrónicos"y habilitado por la "simultaneidad" que la presencialidad habilita.

A partir de la crítica, los autores postulan, desde una posición en las antípodas, una racionalidad fenomenológica del tiempo sostenida en la concepción de que se trata de un fenómeno subjetivo, en consecuencia, relativo y, frente a la monocronía, destacan la condición policrónica.

Ahora bien, en estos "tiempos de pandemia" y virtualidad, nos cuestionamos, ¿cuánto de la racionalidad técnico instrumental dejaremos colar en nuestras propuestas? ¿o será esta una oportunidad para descolonizar el tiempo escolar, para corrernos de las lógicas administrativistas y convertirla en una instancia que abra un "horizonte de posibilidades", que a modo de ensayo, impacte posteriormente en las prácticas docentes y de la enseñanza en la presencialidad?

Respecto del espacio escolar no es posible ignorar, desde el planteo filosófico -Foucault en adelante-, hasta innumerables relatos, las diversas calificaciones que lo caracterizan como "espacio de clausura" y a la escuela como institución de disciplinamiento y encierro que opera un aislamiento preventivo, al que Ariès definió como cuarentena moral. Aislamiento que posibilita las operaciones de diferenciación...(identificación de un límite)... y distinción con el resto, con lo que no es escuela... y que permite una serie de distinciones (interior/ exterior; afuera/adentro). Ahora bien, dice el autor, también la escuela nos enseñó el deseo de salirse, de irse, de circular, de liberarse. Es decir, de reconocer la contracara: encerrar para cuidar, para contener, para educar, para producir...así la escuela es refugio, comunidad...envolver y dejarse envolver, envolverse entre sí, mutuamente, para mejorar la existencia. (Antelo, 2013)

Necesariamente una lectura didáctica impone un pasaje de la institución al aula, significada como espacio físico de alto valor simbólico, estructura arquitectónica y de comunicación, hábitat de sujetos en agrupamientos prefigurados, lugar de encuentro signado por la particular forma que en ella adopte la triádica relación docente, alumno, 
conocimiento; ámbito principal de realización de la escena pedagógica cotidiana (Edelstein, Salit y otras, 2008).

Nos preguntamos, ¿cómo sostener un espacio de contención, de cercanía, de co-construcción colectiva en la virtualidad? ¿Cómo sortear la ausencia de proximidad física? ¿Cómo habitar esos espacios para muchos de nosotros lejanos?

Nos preguntamos, ¿cómo sostener un espacio de contención, de cercanía, de co-construcción colectiva en la virtualidad? ¿Cómo sortear la ausencia de proximidad física? ¿Cómo habitar esos espacios para muchos de nosotros lejanos?

No desconocemos que las tecnologías digitales introducen formas diferentes de organizar el tiempo y el espacio de la enseñanza y de los aprendizajes en la virtualidad. El intercambio con entornos tecnológicos nos plantea una serie de nuevos desafíos. Siguiendo a Lion

(...) resignificar los tiempos del aula más allá de sus fronteras...pensar en propuestas flexibles, diversificadas, en las que haya mayor tolerancia a la espera y mayor lugar para la reflexión en la interacción con Internet, a pesar de su fugacidad....generar espacios compartidos (sincrónicos y asincrónicos)... pensar que los ritmos de la enseñanza y del aprendizaje merecen ser interpretados, a la luz de la influencia tecnológica, en términos de fugacidad, atemporalidad e imprevisibilidad, y redimensionados para favorecer procesos críticos de apropiación del conocimiento. $(2005$, p. 189)

En esa dirección, la noción de itinerarios nos permite pensar en un posible camino, en un orden tentativo, en ciertas relaciones, y en que una propuesta es susceptible de ser modificada, redefinida. De ahí la idea de imaginar diferentes líneas en cierta dirección, desvíos y retornos. Un itinerario debe ser producido a partir de considerar la historia de elaboración y producción específicas de saberes al interior del campo de conocimiento, así como las formas de apropiación, las historias e identidades culturales de los sujetos destinatarios ${ }^{1}$.

En consonancia con estas consideraciones, y ante la condición de una relación docente-alumno separada en el tiempo y en el espacio, intentamos generar recursos y herramientas, hacia la construcción de otro modo de presencia, que desplieguen configuraciones potentes a partir de la convergencia de diversas mediaciones y soportes tecnológicos. Es decir, atender a la lógica del contenido y a la lógica de la interacción, en el particular esfuerzo que demanda articular forma y contenido.

En pos de ello, venimos trabajando en la construcción de una caja de herramientas, que tiene en cuenta, entre otros recaudos, los siguientes: la diversidad de tiempo/tiempos de la conexión y la participación

1 Les destinatarios de la propuesta de enseñanza en la virtualidad que aquí estamos describiendo, se conforma por el alumnado que ya cursó con el equipo de cátedra la asignatura Pedagogía durante el segundo semestre de 2019 constituido por 100 integrantes aproximadamente. 
de les estudiantes; actividades variadas y con distintos propósitos, que se puedan resolver individual o grupalmente; diversidad en los modos de presentar los contenidos; el ofrecimiento de múltiples canales de comunicación con la cátedra; la construcción de herramientas de estudio específicas para el nuevo escenario de aprendizajes.

En pos de ello, venimos trabajando en la construcción de una caja de herramientas, que tiene en cuenta, entre otros recaudos, los siguientes: la diversidad de tiempo/tiempos de la conexión y la participación de les estudiantes; actividades variadas y con distintos propósitos, que se puedan resolver individual o grupalmente; diversidad en los modos de presentar los contenidos; el ofrecimiento de múltiples canales de comunicación con la cátedra; la construcción de herramientas de estudio específicas para el nuevo escenario de aprendizajes.

En el siguiente cuadro incorporamos una breve descripción de cada una de ellas.

Hoja de ruta. Creamos un recorrido para subir al aula virtual y compartir con les estudiantes fechas, actividades, lecturas y mensajes, los cuales constituyen medios para orientar el cursado virtual de la materia.

Podcasts. Ofrecemos un recorrido de audios donde se trabaja algún núcleo conceptual de la propuesta de la cátedra. La importancia de que sea presentado y/o distribuido en varios audios acotados en el tiempo y no uno de larga duración sin cortes, responde a la intención de generar la posibilidad de hacer una pausa, anotar preguntas, -quizás lo que no se entiende del planteo-, y luego continuar escuchando los audios siguientes.

Fichas de cátedra. Constituyen materiales de estudio teórico elaborados por la cátedra a partir de diversos textos que se ponen en diálogo, a modo de entramado de voces, posicionamientos, perspectivas.

Ficha bibliográfica. Herramienta tradicionalmente usada, pero que recuperamos y sugerimos para acompañar y registrar la lectura y el estudio de los textos de la bibliografía en su contexto y tiempo histórico. Ofrecemos un modelo posible para incorporar datos relevantes de cada uno de ellos. 
Y utilizamos los siguientes espacios virtuales para la resolución de actividades

En este punto cabe aclarar que inicialmente planteamos el uso de foros para habilitar espacios de debate e intercambio. No obstante, un problema técnico (que excede nuestras posibilidades de resolución) inhabilitó el uso de este recurso a nivel facultad. Este imprevisto nos impulsó a generar otras alternativas que nos permitieran sostener espacios de participación en el que todes se puedan leer.

Padlet (mural). Un espacio en el cual se pueden compartir diferentes producciones (textos, imágenes, audios, videos, etc.) para que, de alguna manera, todos puedan acceder a ellas. Como actividad inicial, propusimos una consigna que posibilitara recuperar los retratos de docentes que les estudiantes habían realizado en la cátedra de Pedagogía, cursada en el año anterior. La intención era que les estudiantes se imaginaran como futuros docentes en artes y compartieran una breve reflexión acerca de ello.

Glosario. Propusimos una resignificación del sentido original de este recurso (crear y mantener una lista de definiciones, de forma similar a un diccionario), habilitando el espacio para que les estudiantes ingresen textos breves a partir de consignas puntuales. Si bien no genera la posibilidad de intercambios entre los participantes, ofrece la vista de todas las intervenciones realizadas y pone en común las producciones de cada une.

\section{B. SEGUNDO DESAFÍO. CUANDO LA GESTUALIDAD, EL TONO Y LOS MATICES DE LA VOZ ESTÁN AUSENTES: EL VALOR DE LO QUE ESCRIBIMOS}

Cuando se trata de sostener una propuesta formativa en la virtualidad no es posible obviar que

en nuestra labor diaria (leyendo, escuchando, escribiendo) emerge lo cotidiano, la confianza e intimidad, emerge lo colectivo, pero también lo personal, subjetivo e individual, yo-nosotros "del otro lado de la pantalla", nos hacemos presente para un "otro" desde diversas expresiones: sorpresa, de disfrute, de alegría, de reflexión, de duda, de emoción, de ansiedad, etc. Tomamos la palabra desde lo singular, desde lo subjetivo, pero también desde lo 
colectivo, junto a aquellos elementos y lugares que, a veces, se invisibilizan en la tarea "detrás de la pantalla", pero están allí trazando la tarea: intervenimos mientras hacemos las actividades con los hijos, mientras cocinamos, mientras leemos, tomamos algún rico mate, junto algún que otro libro, con música de fondo, las plantas, las mascotas...etc. (Yazyi, 2015)

Es decir, un modo singular de habitar las aulas virtuales, un modo "otro" de "estar ahí", de hacer presente la propuesta. En tal sentido, las actividades, mensajes, recursos, fichas, pretenden generar proximidad. Construir proximidad es una estrategia fundamental en el trabajo virtual, que puede tener efectos en el sentido de "habitar las aulas" en la medida que las producciones sean basadas en una genuina construcción pedagógico-didáctica para generar intercambio. Esto implica crear condiciones de intercambio, construir un estilo de escritura y comunicación que pedagógicamente habilite un lugar a les estudiantes.

En efecto, una manera de habitar, de hacer nuestra y de los otros, el aula virtual, es dialogar mediante la palabra escrita, compartir sensaciones, contrastar miradas en relación a las temáticas, aportar otros recursos, comunicar reflexiones. Leernos resulta una tarea central para poder producir un saber colectivo, que nunca es acabado y que se encuentra en permanente construcción.

\section{Leernos resulta una tarea central para poder producir un sa- ber colectivo, que nunca es acabado y que se encuentra en permanente construcción.}

En ese sentido, nos preguntamos, ¿qué viraje se produce en la comunicación ante el pasaje de la presencialidad a la virtualidad? ¿Qué tipo de narrativas utilizar en la virtualidad? ¿Cómo expresar a través de la palabra escrita el mensaje que queremos transmitir?

Entendemos que la enseñanza es básicamente discursiva, que se sostiene en el lenguaje, esto es: en qué se dice y cómo se dice; en qué se escribe y en qué se lee, que en el encuentro cara a cara se acompaña la palabra hablada con gestos, tonos, intensidades. Se trata de un quehacer que valida ciertos discursos y desautoriza otros, que legitima y deslegitima voces, sujetos y posiciones. Así también la escritura es una práctica social situada y que, por lo tanto

(...) se despliega en contextos comunicativos concretos en función de los cuales se producen ajustes léxicos y de organización estructural orientados a la vez por la imagen que se haya configurado acerca del lector y según el propósito perseguido por quien toma la palabra. (Scarafía y Constenla, 2011, p. 37) 
Algunos autores señalan que, en el contexto de la virtualidad, se establece un particular tipo de relación entre las acciones y los soportes a través de los cuales se estructuran básicamente textos escritos; por lo tanto, atender a las condiciones de escritura resulta ineludible. En vínculo con ello, las autoras antes citadas reconocen que

(...) se produce la emergencia de registros mixtos o híbridos como la "ciberhabla" o "neoescritura" que favorecen la intervención dialógica en los espacios de foro y chat, pero que lo ubican frente a nuevas y desconocidas formas de comunicarse (2011, p.35). (...) La novedad, no radica en el hecho de que la función docente le exija al tutor mediar a través de "tecnologías de la palabra" e interactuar con el alumno, quien a través de ellas aprende, estructura su pensamiento y construye conocimiento; lo que subrayamos es que aun cuando se vuelvan efímeros soportados en la virtualidad, los textos constituyen la materialidad a través de la cual el docente se expresa. (2011, p. 35)

En ese marco, continúan, todo docente que trabaje en la virtualidad habrá de enfrentarse cuanto menos a tres tipos generales de texto: exposición (explicativa y argumentativa) diálogo e instrucción. En nuestro caso hacemos una oferta diversificada de textos que más allá de las tipologías fueron construidos atendiendo, centralmente a encontrar narrativas y soportes que reflejen desde la forma, las intencionalidades y los contenidos puestos en juego.

\section{C. TERCER DESAFÍO, ¿RESIGNIFICAR ESO QUE LLAMAMOS DEVOLUCIÓN, EN LA VIRTUALIDAD?}

Si buscamos el significado de "devolver" encontramos que alude a la acción de "hacer que una persona tenga de nuevo lo que había dejado de tener". Ante esa afirmación nos preguntamos, ¿qué sentidos de este significado se trasladan a la idea de "devolución" en la virtualidad en el marco de una práctica de enseñanza?

\section{¿qué sentidos de este significado se trasladan a la idea de "devolución" en la virtualidad en el marco de una práctica de enseñanza?}

Desde nuestra perspectiva, entendemos que enseñar implica siempre un proceso caracterizado por sucesivas mediaciones, ya que el conocimiento sufre una serie de transformaciones a los efectos de la transmisión: es reinterpretado, parcializado, jerarquizado y transmitido de manera discontinua. Ello vuelve necesario construir puentes entre las estructuras epistemológicas de las disciplinas y las estructuras cognitivas y socio afectivas de los sujetos. 
En el conjunto de intercambios/actividades que se producen en el espacio virtual, las mediaciones favorecen especialmente los procesos de apropiación de saberes de la asignatura y las formas de relación crítica con los conocimientos. Una devolución encarna entonces un entretejido de significados y se convierte en un acto de creación e innovación en tanto es parte de la tarea de enseñanza y es, a través de ella, que la práctica docente se especifica, constituyendo el acontecimiento que organiza la escena en la que se vinculan los sujetos a través del conocimiento, donde se juegan las relaciones intersubjetivas. (Programa de la cátedra Didáctica General, 2020)

En este sentido, construir una devolución es, para nosotras, una manera más de mediar entre eso que les pedimos a los estudiantes que "hagan" y aquello que les estudiantes "entregan" en una actividad.

\section{En este sentido, construir una devolución es, para nosotras, una manera más de mediar entre eso que les pedimos a los estudiantes que "hagan" y aquello que les estudiantes "entre- gan" en una actividad.}

Nos preguntamos, ¿qué cuestiones creemos que son importantes considerar al momento de hacer devoluciones de las resoluciones de actividades que les estudiantes presentan? ¿Cómo expresarlo para que se entienda y no genere confusiones? La respuesta contempla el esfuerzo por labrar formas particulares de construir vínculo pedagógico con el saber, entre estudiantes y docentes. En ese sentido, construimos textos que conjuguen las propias voces de les estudiantes, incorporando a ellas, nuevas reflexiones y saberes que operen no como un "cierre" de la actividad, sino, por el contrario, como parte de una conversación que se sostiene y continúa con renovados énfasis actividad tras actividad. Es éste un modo más de procurar sostener la relación forma-contenido.

\section{EL DESAFÍO DE ARTICULAR FORMA Y CONTENIDO AL ENSE- ÑAR A ENSEÑAR EN LA VIRTUALIDAD}

Decimos "enseñar en la virtualidad" y no "a distancia" ya que asumir la modalidad a distancia requiere de una serie de condiciones que van desde una estructura organizativa a condiciones tecnológicas y formativas que no están garantizadas en este caso. La transmisión pedagógica que proponemos en este momento, apelando a la utilización de dispositivos con los que contamos docentes y estudiantes, se presenta de modo improvisado y urgente -dado el escenario de emergencia- $y$ es por ello que es preciso reconocer su carácter de excepcionalidad en el marco de esta coyuntura y actuar en la inmediatez.

Somos conscientes que el uso de la virtualidad en propuestas de enseñanza, como toda innovación en las prácticas, debe necesariamente sostenerse en coherencia con las teorías a las que adscribimos. Por 
ello, reafirmamos nuestra perspectiva de una didáctica de carácter crítico-propositivo que se aleja de la racionalidad técnico-instrumental que caracterizó la historia de configuración de la disciplina. Ello adquiere particular valor cuando lo que está en juego es precisamente del orden de lo tecnológico.

Decimos "enseñar en la virtualidad" y no "a distancia" ya que asumir la modalidad a distancia requiere de una serie de condiciones que van desde una estructura organizativa a condiciones tecnológicas y formativas que no están garantizadas en este caso.

En clave con ello, recuperamos aportes de numerosos autores, entre ellos, Burbules (2011), quienes sostienen que las tecnologías son prometedoras, pero también riesgosas. Esos riesgos son políticos, subjetivos y también técnicos. Cuando uno decide correrlos, es preciso extender la experiencia de "incertidumbre" que caracteriza a la enseñanza en general (Jackson, 2002), a la que integra herramientas propias de la cultura digital, que nos enfrentan a muy diversos imprevistos.

Desde este marco de ideas, no exento de instancias de debate al interior del equipo de cátedra, de inseguridades y vacilaciones, nos desafiamos a la construcción de caminos posibles para repensar la relación forma-contenido en el aula virtual. Compartimos hasta aquí preguntas, inquietudes, y los particulares modos desde los cuales venimos reconstruyendo una propuesta inicialmente pensada para la presencialidad.

Compartimos hasta aquí preguntas, inquietudes, y los particulares modos desde los cuales venimos reconstruyendo una propuesta inicialmente pensada para la presencialidad.

Sostenemos la convicción que estos tiempos inusitados invitan a convocar la imaginación y elaborar propuestas en diversos sentidos "inéditas" que trascienden la presencialidad, en las cuales las aulas virtuales deberán convertirse en "lugares habitables", es decir, "espacios para habitar" en los que no nos sintamos tan solos.

Finalmente, y en clave con ello, nos preguntamos si será posible en la virtualidad aceptar el convite de Recalcati (2016) en la dirección de que una hora de clase cambie una vida, le de al destino otra dirección y permita toparse con lo inesperado, con lo maravilloso. 


\section{BIBLIOGRAFIA}

Antelo, E. (2013). "Variaciones sobre el espacio escolar." En: Baquero, R.,Diker,G. y Frigerio, G. (2013) Las formas de lo escolar. Buenos Aires: Del Estante.

Berisso, D. (2015). ¿Qué clase de dar es el dar clase?. Alteridad, donación y contextualidad. Buenos Aires: Antropofagia.

Burbules, N. (2011). Reportaje: "El aprendizaje ubicuo es hacer que el aprendizaje sea una experiencia más distribuida en el tiempo y el espacio". Instituto Nacional de Planeamiento de la Educación. Buenos Aires, UNESCO.

Dussel, I; Caruso, M. (1999). "La invención del aula. Una genealogía de las formas de enseñar". Buenos Aires: Santillana.

Edelstein, G.; Salit, C.; Domjan, G. y Gabbarini P. (2014). “Enseñar en procesos de residencia: Alternativas Didácticas. Ponencia: “VI Jornadas Nacionales de Prácticas y residencias en la Formación Docente... Córdoba.

Edelstein, G.; Salit, C.; Domján, G., Gabbarini, P. (2008). "Módulo 2: Práctica Docente. Programa de capacitación docente continua a distancia". Buenos. Aires, UNLA.

Edwards, D. y Mercer, N. (1994). "El conocimiento compartido. El desarrollo de la comprensión en el aula". Barcelona: Paidós.

Edwards, V. (1988). “El conocimiento escolar como lógica particular de apropiación y alienación". En: Torres, R. M. Remedi, E. Landesmann, M. Edwards, V. Curriculum, maestro y conocimiento. Universidad Autónoma Metropolitana. México, UNAM-Xochimilco.

Jackson, P. (2002). “Práctica de la enseñanza”. Buenos Aires: Amorrortu.

Lion, C. (2005)."Nuevas maneras de pensar tiempos, espacios y sujetos". En Litwin, E. Tecnologías educativas en tiempos de internet. $1 \mathrm{ra}$ Ed. Buenos Aires: Amorrortu.

Litwin, E. (1995). “El campo de la Didáctica: la búsqueda de una nueva agenda"En: Corrientes Didácticas Contemporáneas. Buenos Aires: Paidós.

Poggi, M. (1999). "Del tiempo escolar". En: Frigerio, G, Poggi, M y Korinfeld, D. (1999) Construyendo un saber sobre el interior de la escuela. Buenos Aires: Ediciones Novedades Educativas.

Programa de Didáctica General (2020). Facultad de Artes, Universidad Nacional de Córdoba.

Recalcati, M. (2016). “La hora de clase. Por una erótica de la enseñanza". Bar- 
Salit, C. (2011). “Enseñar didáctica. Sentidos construidos desde una propuesta de formación situada". Ponencia concurso.

Scarafía, S. y Constenla, M. (2011). “Docencia universitaria virtual y demandas de escritura: Un nuevo desafío de alfabetización". Revista VEsC -Año 2- Número 3- pp. 29-42.

Terigi, F. (2008). “El saber pedagógico frente a la crisis de la monocronía”. En Frigerio, G. y Diker, G. (Comp.). Educar: saberes alterados. Buenos Aires: CLACSO Coediciones - Del estante editorial.

Yazyi, M. (2015). “Reflexión final foro ¿Desde qué lugar/es (en el mundo) tomó la palabra? Discutir sentidos del equipo coordinadores y tutores. Materia Prácticas de Enseñanza con TIC, Especialización Superior en Educación Primaria y TIC, Instituto Nacional de Formación Docente, Ministerio de Educación de la Nación. Argentina. 